\title{
Age-related differences in breast cancer mortality according to race/ethnicity, insurance, and socioeconomic status
}

Yazmin San Miguel', Scarlett Lin Gomezz ${ }^{2,3}$, James D. Murphy', Richard B. Schwab ${ }^{1}$, Corinne McDaniels-Davidson ${ }^{4}$, Alison J. Canchola ${ }^{2}$, Alfredo A. Molinolo ${ }^{1}$, Jesse N. Nodora ${ }^{1,5}$ and Maria Elena Martinez ${ }^{1,5^{*}}$ (iD

\begin{abstract}
Background: We assessed breast cancer mortality in older versus younger women according to race/ethnicity, neighborhood socioeconomic status (nSES), and health insurance status.

Methods: The study included female breast cancer cases 18 years of age and older, diagnosed between 2005 and 2015 in the California Cancer Registry. Multivariable Cox proportional hazards modeling was used to generate hazard ratios (HR) of breast cancer specific deaths and 95\% confidence intervals (Cl) for older (60+ years) versus younger ( $<60$ years) patients separately by race/ethnicity, nSES, and health insurance status.

Results: Risk of dying from breast cancer was higher in older than younger patients after multivariable adjustment, which varied in magnitude by race/ethnicity (P-interaction<0.0001). Comparing older to younger patients, higher mortality differences were shown for non-Hispanic White $(H R=1.43 ; 95 \% \mathrm{Cl}, 1.36-1.51)$ and Hispanic women $(H R=$ 1.37; $95 \% \mathrm{Cl}, 1.26-1.50)$ and lower differences for non-Hispanic Blacks ( $\mathrm{HR}=1.17 ; 95 \% \mathrm{Cl}, 1.04-1.31)$ and Asians/ Pacific Islanders ( $\mathrm{HR}=1.15 ; 95 \% \mathrm{Cl}, 1.02-1.31)$. HRs comparing older to younger patients varied by insurance status (P-interaction< 0.0001), with largest mortality differences observed for privately insured women $(\mathrm{HR}=1.51 ; 95 \% \mathrm{Cl}$, 1.43-1.59) and lowest in Medicaid/military/other public insurance ( $H R=1.18 ; 95 \% \mathrm{Cl}, 1.10-1.26)$. No age differences were shown for uninsured women. HRs comparing older to younger patients were similar across nSES strata.

Conclusion: Our results provide evidence for the continued disparity in Black-White breast cancer mortality, which is magnified in younger women. Moreover, insurance status continues to play a role in breast cancer mortality, with uninsured women having the highest risk for breast cancer death, regardless of age.
\end{abstract}

Keywords: Mortality, Younger and older age, Breast cancer

\section{Background}

According to American Cancer Society, the 10-year probability of developing breast cancer increases with age, from $0.5 \%$ in women 30 years of age to $3.9 \%$ in those age 70 and the median age of diagnosis is 62 [1]. In 2015,

\footnotetext{
* Correspondence: e8martinez@ucsd.edu

${ }^{1}$ Moores Cancer Center, University of California San Diego, La Jolla, CA, USA ${ }^{5}$ Department of Family Medicine and Public Health, University of California San Diego, La Jolla, CA, USA

Full list of author information is available at the end of the article
}

$58.0 \%$ of all incident breast cancers in the United States (U.S.) occurred in women over the age of 60 [2]. With a rising number of older women in the U.S., understanding the breast cancer burden, including survival outcomes in these women is important. While different age cut-offs are used to define younger versus older patients, it is well recognized that women less than 40 years of age are more likely to develop breast cancer with more aggressive subtype and worse clinicopathological features [1,3]. Findings from published studies report differences in breast cancer

(C) The Author(s). 2020 Open Access This article is licensed under a Creative Commons Attribution 4.0 International License, which permits use, sharing, adaptation, distribution and reproduction in any medium or format, as long as you give appropriate credit to the original author(s) and the source, provide a link to the Creative Commons licence, and indicate if changes were made. The images or other third party material in this article are included in the article's Creative Commons licence, unless indicated otherwise in a credit line to the material. If material is not included in the article's Creative Commons licence and your intended use is not permitted by statutory regulation or exceeds the permitted use, you will need to obtain permission directly from the copyright holder. To view a copy of this licence, visit http://creativecommons.org/licenses/by/4.0/ The Creative Commons Public Domain Dedication waiver (http://creativecommons.org/publicdomain/zero/1.0/) applies to the data made available in this article, unless otherwise stated in a credit line to the data. 
mortality and survival by age, showing that women diagnosed with breast cancer at less than 40 years of age have a lower survival than older patients [4-6]. Studies focused on breast cancer mortality have also reported that younger compared to older breast cancer patients have higher breast cancer mortality, regardless of age-cut off $[7,8]$. While a wealth of published data exists on factors that impact breast cancer mortality, including race/ethnicity, socioeconomic status, and other sociodemographic factors [9-11], limited research exists on whether associations vary by age at diagnosis.

Few studies have been published on factors associated with breast cancer mortality in older women when compared to younger patients, with varying age cut-offs [1217]. Published results show that older women with worse breast cancer health outcomes are more likely to be racial/ethnic minority women, have a lower sociodemographic status, and have no health insurance $[13,15$, 17]. Under-treatment and/or more comorbidities in older women compared to younger women could be reasons for the observed higher breast cancer mortality in these women $[12,13,16]$. It has been reported that as women age, they are less likely to pursue or be offered aggressive treatment [12]. To our knowledge, studies of age differences in survival have not considered potential heterogeneity by sociodemographic factors, which would aid in better understanding breast cancer outcomes.

Using data from the population-based California Cancer Registry (CCR), our study assessed breast cancer mortality differences between younger (age 18-59) and older (age 60 and above) breast cancer patients according to race/ ethnicity, health insurance, and socioeconomic status, while controlling for patient and clinical variables.

\section{Methods}

\section{Study population}

We obtained information from the CCR for female California residents ages 18 years and older at diagnosis, who were diagnosed with a first, primary invasive breast cancer [International Classification of Disease for Oncology, 3rd Edition, (ICD-O-3) site codes C50.0-50.9] during January 1, 2005 through December 31, 2015 ( $n=$ $219,266)$. Patients were excluded from the analysis hierarchically as follows: diagnosis by death certificate or autopsy only $(n=889)$ or diagnosis not microscopically confirmed $(n=1698)$; ICD-O-3 histologic type other than: 8000, 8001, 8010, 8020,8022, 8050, 8140, 8201, $8211,8230,8255,8260,8401,8453,8480,8481,8500-$ 8525 , or $8575(n=3654)$; tumor size missing because unknown $(n=8347)$, no tumor noted $(n=510)$, microscopic $(n=2250)$, diffuse $(n=608)$, or mammographic diagnosis only $(n=59)$; age $<60$ insured by Medicare $(n=513)$; no follow-up $(n=269)$; residential address that was uncertain or not geocodable $(n=6292)$. The study included 192,932 patients, of whom 94,076 were younger (age 18-59) and 98,856 were older (age 60 and above, up to age 109) patients.

\section{Data acquisition}

Data from the CCR, mostly derived from the patient's medical record, were used to obtain age at diagnosis, marital status, residential address at diagnosis, stage at diagnosis, tumor size (in centimeters), lymph node involvement, histology, grade (I, II, III/IV, or unknown), and hormone receptor [estrogen receptor (ER), progesterone receptor (PR), and human epidermal growth factor receptor 2 (HER2)] status. The CCR followed patients for vital status, from linkage with vital records, to December 31, 2015 for this study.

For the variables of interest in the present report, we used data from the medical record to classify race/ethnicity as non-Hispanic White (NHW), non-Hispanic Black, Hispanic, Asian/Pacific Islander (API), or other/unknown; and primary and secondary source of payment were used to classify insurance status, as private only, Medicare only/ Medicare + private, any Medicaid/military/other public, no insurance, and unknown; in the CCR, payer status is coded based on the most extensive insurance type across the diagnosis to treatment continuum. We used a multicomponent measure of neighborhood socioeconomic (nSES), based on patients' residential census block group at diagnosis. This measure incorporated the 2000 U.S. Census (for cases diagnosed in 2005) and the 2006-2010 American Community Survey data (for cases diagnosed in 2006 and forward) on education, occupation, unemployment, household income, poverty, rent, and house values $[18,19]$. Each patient was assigned a nSES quintile, based on the distribution of socioeconomic status across census block groups in California.

\section{Statistical analysis}

Given the lack of standard for categorizing younger and older breast cancer patients, we used the median age of the study population as a cut-off ( 60 years); younger women included those age 18-59 and older included 60+ years. Differences in mortality for older and younger women were examined by two methods. First, comparisons were made between older and younger patients stratified by race/ethnicity, insurance status, and nSES, with younger women as the reference group. Next, models were stratified by age and comparisons were made between race/ethnicity (nonHispanic White as the reference group), insurance status (private insurance as the reference group), and nSES quintiles (5th quintile as the reference group).

Descriptive statistics were calculated and reported as percentages for categorical data and means with standard deviation for continuous variables. Covariates were shown overall and for younger and older women. Covariates 
examined included: age (continuous and categorical), race/ ethnicity, marital status, insurance status, nSES, whether the patient was seen at one or more of the National Cancer Institute-designated Cancer Centers in California (NCICC) for her breast cancer, American Joint Committee on Cancer (AJCC) stage at diagnosis, tumor subtype, lymph node involvement, tumor size, tumor grade, and tumor histology.

Follow-up time was calculated as the number of days between the date of diagnosis and date of death from breast cancer (ICD $9 / 10=174 / C 50$ ), the date of death from another cause, the date of last follow-up (i.e., last known contact), or the study end date (12/31/2015). There were 599 deceased patients with an unknown cause of death which were excluded from all models. Cox proportional hazards regression was used to estimate breast cancer specific hazard rate ratios (HR) and corresponding 95\% confidence intervals (CI). Adjusted models were stratified by AJCC stage and adjusted for age at diagnosis (continuous), year of diagnosis (continuous), race/ethnicity, marital status, insurance status, nSES, whether the patient was seen at one or more of the NCICC in California for her breast cancer, tumor subtype, lymph node involvement, tumor size, tumor grade, and tumor histology. Fully adjusted models were additionally adjusted for clustering by block group, using a sandwich estimator of the covariance structure that accounts for intracluster dependence. The proportional hazards assumption was tested by examining the correlation between time and scaled Schoenfeld residuals for all covariates. The proportional hazards assumption was violated for AJCC stage at diagnosis, tumor subtype, and tumor grade. Stage was included as an underlying stratifying variable in the fully adjusted Cox regression models reported here, which allowed the baseline hazards to vary by stage. Additionally, stratifying the Cox model by tumor subtype and tumor grade did not meaningfully change the HR for the main effect of age, so these factors were simply adjusted for in fully adjusted models. Wald Type 3 tests for interaction between age group $(18-59,60+)$ and race/ethnicity, insurance status, and nSES and were computed using crossproduct terms, in models adjusted for all statistically significant $(p<0.05)$ interactions with age group (race/ethnicity, marital status, insurance status, NCICC, tumor subtype, and lymph node involvement). Wald tests for trend across nSES quintiles were computed using quintile number as an ordinal variable. All statistical tests were carried out using SAS software version 9.3 (SAS Institute).

\section{Results}

Of the total population $(n=192,932), 94,076(48.7 \%)$ were diagnosed under the age 60 and 98,856 (51.2\%) were aged 60 and older. As noted in Table 1, approximately $60 \%$ of the population was NHW, $40 \%$ was married, $26 \%$ was from the highest socioeconomic neighborhood, and 58\% had private insurance. Examining clinical factors, $48 \%$ of total patients were diagnosed with stage I breast cancer, $65 \%$ had hormone receptor-positive (ER positive or PR positive)/ HER2-negative tumor subtype, $66 \%$ were negative for lymph node involvement, $35 \%$ had a tumor size of $1-2 \mathrm{~cm}$, $42 \%$ had a tumor grade of II, $79 \%$ had a ductal histology, and $12 \%$ received care at a NCI-designated Cancer Center.

Table 2 shows the multivariable-adjusted breast cancer specific mortality for older versus younger women according to race/ethnicity, health insurance, and nSES. Overall, older breast cancer patients had a higher risk of dying from breast cancer than younger patients $(\mathrm{HR}=$ 1.35; 95\% CI, 1.29-1.40). HRs (95\% CIs) comparing older to younger women were: $1.43(1.36-1.51)$ for NHWs; 1.37 (1.26-1.50) for Hispanics; 1.17 (1.04-1.31) for non-Hispanic Blacks; and 1.15 (1.02-1.31) for APIs. A higher risk of dying was shown for older vs. younger patients for women with private insurance $(H R=1.51$; 95\% CI, 1.43-1.59) and for those with any Medicaid/ military/other public insurance $(\mathrm{HR}=1.18 ; 95 \% \mathrm{CI}$, 1.10-1.26). No significant differences by age were shown for uninsured patients. HRs by age across nSES quintiles ranged from 1.30 (95\% CI, 1.19-1.41) in the third quintile to $1.42(95 \% \mathrm{CI}, 1.29-1.56)$ in fifth quintile.

Table 3 shows HRs stratified for older and younger women separately, according to race/ethnicity, insurance status, and nSES. Compared to NHWs, non-Hispanic Blacks had a higher risk of dying regardless of age group, with higher HRs for younger $(\mathrm{HR}=1.36$; $95 \% \mathrm{CI}, 1.25$ 1.48) than older ( $\mathrm{HR}=1.11 ; 95 \% \mathrm{CI}, 1.01-1.22)$ patients. API women had lower risk of dying compared to NHWs in both age groups: $\mathrm{HR}(95 \% \mathrm{CI})$ was $0.88(0.82-0.95)$ in younger and $0.77(0.70-0.84)$ in older patients. No mortality difference was observed for Hispanics compared to NHWs in either age group. In younger women, compared to patients with private health insurance, a higher risk of breast cancer mortality was observed in women with any Medicaid/military/other public insurance $(\mathrm{HR}=1.49$; $95 \%$ $\mathrm{CI}, 1.41-1.58)$ and in those with no insurance $(\mathrm{HR}=1.96$; 95\% CI, 1.65-2.32). As noted in the Methods, younger patients with Medicare insurance were excluded from the analysis. A higher risk of mortality was shown among older women with any Medicaid/military/other public insurance $(\mathrm{HR}=1.13 ; 95 \% \mathrm{CI}, 1.06-1.21)$ and those with no insurance $(\mathrm{HR}=1.57 ; 95 \% \mathrm{CI}, 1.22-2.03)$, as compared to privatelyinsured patients. No difference was observed for Medicare only or Medicare plus private insurance in the older group. In both younger and older women, breast cancer mortality risk decreased with increasing nSES quintile $(P$-trend $=<0.0001$ for younger and $<0.0001$ for older patients).

Recognizing that very young breast cancer patients $(<40$ years of age) are more likely to have aggressive tumor subtypes or to have germline mutations [20], resulting in higher mortality compared to older patients, we conducted 
Table 1 Patient demographic and clinical characteristics for younger (18-59 years) and older (60+ years) age at breast cancer diagnosis, California, 2005-2015

\begin{tabular}{llllll}
\hline & All & & Younger (18-59) & Older (60+) \\
\hline Total Number of Patients & 192,932 & 100.0 & 94,076 & 100.0 & 98,856 \\
Age (yrs) Mean (SD) & $60.2(13.7)$ & & $48.8(7.3)$ & & $71.2(8.3)$ \\
Age category & & & & & \\
18-39 & 10,785 & 5.6 & 100.785 & 11.5 & - \\
$40-49$ & 35,297 & 18.3 & 35,297 & 37.5 & - \\
$50-59$ & 47,994 & 24.9 & 47,994 & 51.0 & - \\
$60-69$ & 49,225 & 25.5 & - & - & 49,225 \\
$70-79$ & 31,540 & 16.3 & - & - & 31,540 \\
$80+$ & 18,091 & 9.4 & - & - & 31.9 \\
\end{tabular}

\section{Race/ethnicity}

Non-Hispanic White

116,534

60.4

49,107

52.2

67,427

68.2

Non-Hispanic Black

12,014

6.2

6348

6.7

5666

5.7

Hispanic

22,305

14,058

14.2

Asian/Pacific Islander

15,242

10,629

10.8

Other/unknown

25,871

1.1

1074

16.2

1076

1.1

\section{Marital status}

Married

77,754

40.3

31,324

33.3

46,430

47.0

Unmarried

55.9

59,344

63.1

3408

48,464

49.0

Unknown

7370

3.8

3.6

3962

4.0

Neighborhood (block group) statewide SES quintile

1st (lowest)
2nd
3rd
4th
5th (highest)
Insurance status
Private only
Medicare only or Medicare+Private
Any Medicaid/Military/Other public
No insurance
Unknown

23,702

33,174

12.3

17.2

39,136

45,882

20.3

23.8

51,038

112,154

40,789

33,225

1598

5166

170,509

88.4

11.6

Yes

22,423

91,898

68,825

22,492

7249

2468

125,240

58.1

21.1

17.2

0.8

2.7

1.3

64.9

12,174
15,787
18,584
22,231
25,300

71,611

${ }^{a}$

18,671

1085

2709

80,161

13,915

39,390

36,785

13,251

3609

1041

56,822

41.9
39.1
14.1
3.8
1.1

52,508

32,040

9241

3640

1427

68,418

69.2 
Table 1 Patient demographic and clinical characteristics for younger (18-59 years) and older (60+ years) age at breast cancer diagnosis, California, 2005-2015 (Continued)

\begin{tabular}{|c|c|c|c|c|c|c|}
\hline \multirow[b]{2}{*}{ ER+, PR+//HER2+ } & \multicolumn{2}{|l|}{ All } & \multicolumn{2}{|c|}{ Younger (18-59) } & \multicolumn{2}{|c|}{ Older (60+) } \\
\hline & 19,630 & 10.2 & 11,808 & 12.6 & 7822 & 7.9 \\
\hline ER-, PR-//HER2+ & 9023 & 4.7 & 5467 & 5.8 & 3556 & 3.6 \\
\hline Triple negative & 19,836 & 10.3 & 11,280 & 12.0 & 8556 & 8.7 \\
\hline Unclassified & 19,203 & 10.0 & 8699 & 9.2 & 10,504 & 10.6 \\
\hline \multicolumn{7}{|c|}{ Lymph node involvement } \\
\hline Negative & 126,915 & 65.8 & 56,446 & 60.0 & 70,469 & 71.3 \\
\hline Positive & 64,045 & 33.2 & 36,933 & 39.3 & 27,112 & 27.4 \\
\hline Unknown & 1972 & 1.0 & 697 & 0.7 & 1275 & 1.3 \\
\hline \multicolumn{7}{|l|}{ Tumor size (cm) } \\
\hline $0.10 \leq$ tumor $\leq 0.50$ & 13,488 & 7.0 & 6275 & 6.7 & 7213 & 7.3 \\
\hline $0.50<$ tumor $\leq 1.00$ & 31,342 & 16.2 & 12,706 & 13.5 & 18,636 & 18.9 \\
\hline $1.00<$ tumor $\leq 2.00$ & 67,792 & 35.1 & 31,674 & 33.7 & 36,118 & 36.5 \\
\hline $2.00<$ tumor $\leq 5.00$ & 64,694 & 33.5 & 34,472 & 36.6 & 30,222 & 30.6 \\
\hline$>5.00$ & 15,616 & 8.1 & 8949 & 9.5 & 6667 & 6.7 \\
\hline \multicolumn{7}{|l|}{ Grade } \\
\hline Grade I & 43,518 & 22.6 & 17,726 & 18.8 & 25,792 & 26.1 \\
\hline Grade II & 80,909 & 41.9 & 37,234 & 39.6 & 43,675 & 44.2 \\
\hline Grade III/IV & 61,205 & 31.7 & 35,688 & 37.9 & 25,517 & 25.8 \\
\hline Unknown & 7300 & 3.8 & 3428 & 3.6 & 3872 & 3.9 \\
\hline \multicolumn{7}{|l|}{ Histology } \\
\hline Ductal & 151,631 & 78.6 & 76,477 & 81.3 & 75,154 & 76.0 \\
\hline Lobular & 31,554 & 16.4 & 13,564 & 14.4 & 17,990 & 18.2 \\
\hline Other & 9747 & 5.1 & 4035 & 4.3 & 5712 & 5.8 \\
\hline
\end{tabular}

${ }^{a}$ Medicare-insured patients $<60$ years of age were excluded

analyses excluding women less than 40 years of age. The fully adjusted HR (95\% CI) comparing women 60 years of age and older to those less than 60 years was 1.35 (1.30$1.41)$, indicating no difference in the magnitude of the association compared to our main analysis including younger women. Results of analyses for race/ethnicity, health insurance, and nSES stratified by age were not materially different after excluding women less than 40 years of age than those including these younger patients (data not shown).

\section{Discussion}

To our knowledge, there are no published reports on differences in breast cancer mortality between older compared to younger women according to sociodemographic characteristics, such as race/ethnicity, insurance status, and nSES. As such, this study contributes to the limited literature, showing that breast cancer patients 60 years of age and older had higher breast cancer mortality risk compared to those less than 60 years, with largest differences seen among NHW and Hispanic women, and among women with private insurance.
Our analyses assessed age-related differences in breast cancer mortality using two approaches. The first involved examining mortality differences in older versus younger patients within racial/ethnic, insurance, and nSES groups. In the second approach, we assessed differences across race/ethnicity, insurance status, and nSES among younger and older patients. Results of the first approach showed differential age effects by race/ethnicity and insurance status, but not by nSES. Although older women were at higher risk of dying from breast cancer compared to younger women across all racial/ ethnic groups, differences were smaller for non-Hispanic Black and API patients than for NHWs and Hispanics. For insurance status, age-related mortality differences were more pronounced among privately-insured patients and no differences were shown for uninsured women. Finally, in regard to nSES, mortality differences between older and younger women were not highly variable.

In the second approach, comparisons across race/ethnicity, insurance status, and nSES within younger and older age groups showed mortality risk patterns that differed between the two age groups. Compared to NHWs, 
Table 2 Breast cancer specific hazard ratios comparing older to younger age at diagnosis, stratified by race/ethnicity, insurance status, and neighborhood (block group) statewide SES quintile, California, 2005-2015

\begin{tabular}{|c|c|c|c|c|}
\hline & $\begin{array}{l}\text { Younger }(18-59) \\
\text { No. deaths due to } \\
\text { breast cancer }\end{array}$ & $\begin{array}{l}\text { Older }(60+) \\
\text { No. deaths due to } \\
\text { breast cancer }\end{array}$ & $\begin{array}{l}\mathrm{HR}(95 \% \mathrm{Cl})^{\mathbf{a}} \\
\text { Older vs. Younger } \\
\text { (referent) }\end{array}$ & $\begin{array}{l}\text { HR }(95 \% \text { Cl) } \\
\text { Older vs. Younger } \\
\text { (referent) }\end{array}$ \\
\hline Overall & 7058 & 7706 & $1.14(1.10-1.18)$ & $1.35(1.29-1.40)$ \\
\hline \multicolumn{5}{|l|}{ Race/ethnicity } \\
\hline Non-Hispanic White & 3309 & 5199 & $1.29(1.24-1.35)$ & $1.43(1.36-1.51)$ \\
\hline Non-Hispanic Black & 926 & 682 & $0.91(0.83-1.01)$ & $1.17(1.04-1.31)$ \\
\hline Hispanic & 1892 & 1158 & $1.03(0.96-1.11)$ & $1.37(1.26-1.50)$ \\
\hline Asian/Pacific Islander & 873 & 628 & $1.15(1.04-1.27)$ & $1.15(1.02-1.31)$ \\
\hline Other/unknown & 58 & 39 & $0.75(0.50-1.13)$ & $0.56(0.32-0.97)$ \\
\hline \multicolumn{5}{|l|}{ Insurance status ${ }^{c}$} \\
\hline Private only & 4258 & 2606 & $1.18(1.12-1.24)$ & $1.51(1.43-1.59)$ \\
\hline Any Medicaid/Military/Other public & 2360 & 1585 & $0.87(0.82-0.93)$ & $1.18(1.10-1.26)$ \\
\hline No insurance & 169 & 76 & $0.96(0.74-1.26)$ & $1.05(0.78-1.43)$ \\
\hline Unknown & 271 & 174 & $0.79(0.65-0.95)$ & $1.18(0.96-1.45)$ \\
\hline \multicolumn{5}{|l|}{$\begin{array}{l}\text { Neighborhood (block group) } \\
\text { statewide SES quintile }\end{array}$} \\
\hline 1st (lowest) & 1375 & 1228 & $1.01(0.93-1.09)$ & $1.35(1.23-1.48)$ \\
\hline 2nd & 1482 & 1595 & $1.07(0.99-1.15)$ & $1.24(1.14-1.36)$ \\
\hline $3 r d$ & 1509 & 1649 & $1.09(1.02-1.17)$ & $1.30(1.19-1.41)$ \\
\hline 4th & 1451 & 1670 & $1.19(1.11-1.28)$ & $1.43(1.31-1.56)$ \\
\hline 5th (highest) & 1241 & 1564 & $1.37(1.27-1.48)$ & $1.42(1.29-1.56)$ \\
\hline
\end{tabular}

HR Hazard ratio, Cl Confidence interval, No. Number

${ }^{\text {a }}$ Adjusted for year at diagnosis

${ }^{b}$ Stratified by AJCC stage and adjusted for year of diagnosis, marital status, race/ethnicity (in models not stratified by this), insurance status (in models not stratified by this), nSES (in models not stratified by this), lymph node involvement, tumor subtype, tumor size, tumor grade, tumor histology, NCl-designated cancer center and clustering by block group

${ }^{c}$ Medicare-insured patients $<60$ years of age were excluded

non-Hispanic Black women had higher risk of dying regardless of age group, although the HR was higher in magnitude in younger than older patients. The opposite pattern was observed for APIs who had lower risk of dying compared to NHWs. These findings are consistent with the well-established Non-Hispanic Black-White [1, 21] and API-White [1] mortality disparities previously reported and confirms the consistency of these patterns among older and younger women. The more pronounced Black-White survival difference in younger than older patients may reflect more aggressive disease diagnosed among younger non-Hispanic Black women, and warrants further study $[1,22]$. No difference in mortality was observed between Hispanics and NHWs in either age group. Finally, results for nSES showed that patients residing in lower socioeconomic neighborhoods had higher breast cancer mortality compared to those in higher socioeconomic neighborhoods, regardless of age.

Differences across insurance status in younger women showed that compared to privately-insured women, those in other insurance groups had a higher risk of dying, with the highest risk shown in uninsured patients. Of note, younger patients with Medicare insurance were excluded since this group would likely include patients with worse prognosis than those in the older group, complicating the older vs. younger Medicare comparisons. Medicaid/publicly insured patients and uninsured patients had higher mortality compared to those with private insurance regardless of age group although the HRs were higher for younger women. These results suggest that health insurance plays an important role in explaining disparities in breast cancer mortality, as has been noted in the literature $[23,24]$, and that these disparities are somewhat more pronounced in younger women. Higher mortality in Medicaid patients may be due to challenges with Medicaid insurance processes, whereby patients do not get access to Medicaid insurance until their diagnosis of breast cancer is established. Results of some studies suggest differences in treatment for Medicaid patients compared to privately insured patients [25, 26], with Medicaid patients being less likely to receive more aggressive treatment [26], lessening with age [27], which could be due to older patients qualifying for Medicare. Our results draw similar conclusions to these published reports. When compared to private 
Table 3 Breast cancer specific hazard ratios stratified by age for race/ethnicity, health insurance, and neighborhood socioeconomic status, California, 2005-2015

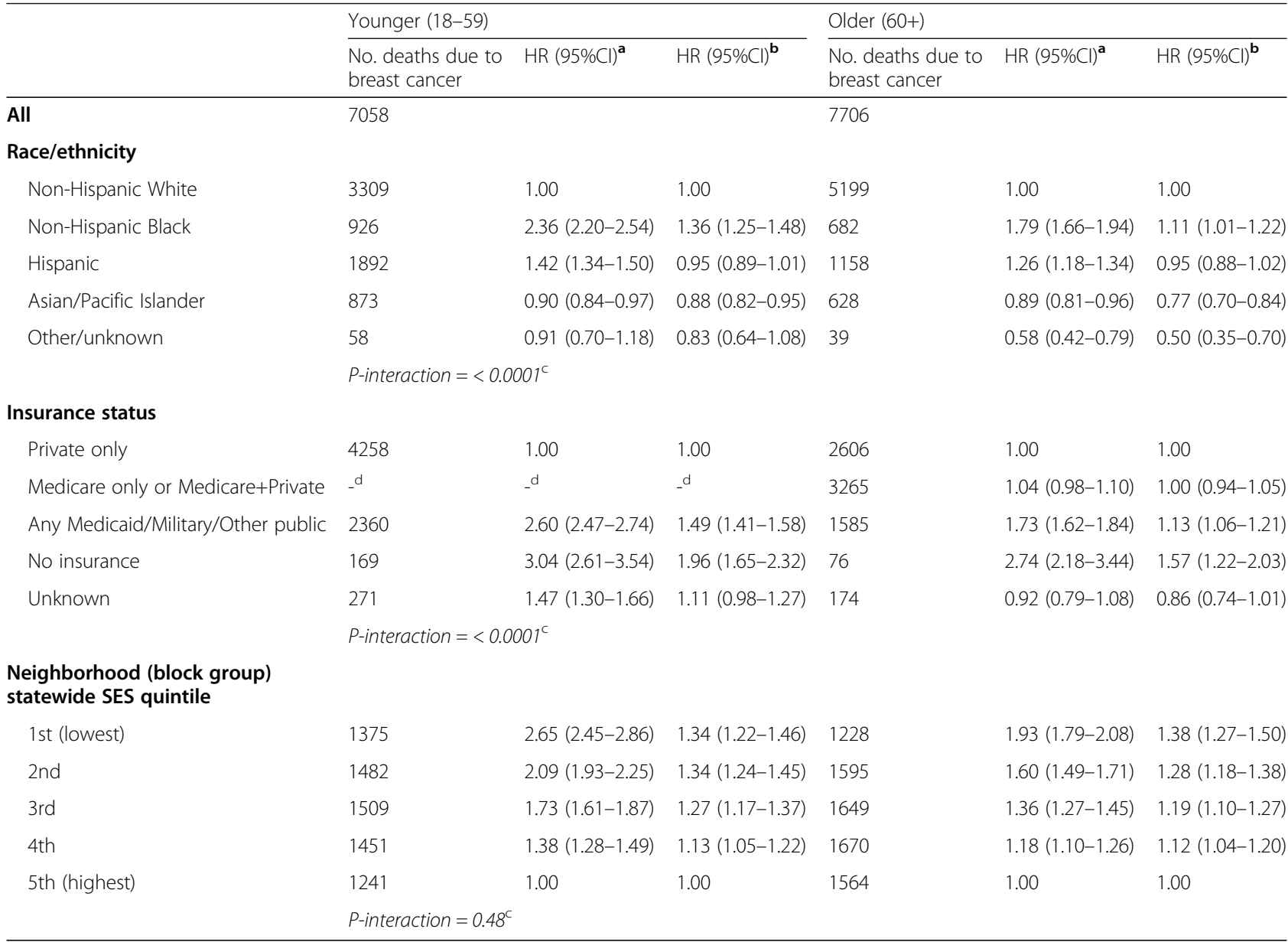

HR Hazard ratio, Cl Confidence interval, No. Number

${ }^{a}$ Adjusted for age at diagnosis and year at diagnosis

b Stratified by AJCC stage and adjusted for age of diagnosis, year of diagnosis, marital status, race/ethnicity, insurance status, nSES, lymph node involvement,

tumor subtype, tumor size, tumor grade, tumor histology, NCl-designated cancer center and clustering by block group

c $P$ for interaction between age group (younger and older) and race/ethnicity, insurance status, or nSES from a model that included all significant interactions with age group

d Medicare-insured patients $<60$ years of age were excluded

insurance, patients with Medicaid had higher mortality, with higher risk in younger than older patients. Additional research is needed to disentangle age differences in the relationship of insurance status on breast cancer mortality.

Findings of our study need to be put into context of limitations. Although results are based on populationbased data covering the entire state of California, given the scarcity of published data on the age-related differences in mortality, these need further validation in other population-based settings. Further, our survival analyses were adjusted for important clinical characteristics. Importantly, we are unable to account for comorbidities because they are not collected as part of the cancer registry. As such, our findings could be subject to residual confounding from incomplete treatment and comorbidity data in the cancer registry [28], which may be especially relevant when comparing older and younger patients. In addition, although it is likely that very young women ( $<40$ years) have a higher risk of mortality than older women [3], which could affect the results of our study, excluding this younger group from the analysis had no appreciable effect on the observed mortality measures. We emphasize that due to limited data on this topic, future population-based studies with more detailed treatment, clinical comorbidity data than those available in the registry are needed to validate our findings and potentially explore mechanisms associated with our observed age-related mortality differences.

\section{Conclusions}

Results from our population-based study show that older breast cancer patients have higher risk of dying from 
breast cancer compared with younger women, with differences more pronounced for NHW, Hispanic, and privately insured women. The prominent Black-White mortality disparity among younger women warrants further study of whether biology, access to treatment, or other factors are driving the particularly poor survival among young non-Hispanic Black women. Health insurance plays an important role in in explaining age-related differences in breast cancer mortality, with greater disparities shown between privately- and non-privatelyinsured patients in younger than older patients.

\section{Abbreviations}

AJCC: American Joint Committee on Cancer; API: Asian/Pacific Islander; CCR: California Cancer Registry; Cl: Confidence interval; ER: Estrogen receptor; HER2: Human epidermal growth factor receptor 2; HR: Hazard ratio; ICD-O3: International Classification of Disease for Oncology, 3rd Edition; NCICC: National Cancer Institute-designated Cancer Centers in California; NHW: Non-Hispanic White; nSES: Neighborhood socioeconomi status; PR: Progesterone receptor; U.S: United States

\section{Acknowledgements}

We would like to thank Valesca Largaespada for her contribution in the manuscript preparation. This work was presented at the American Association of Cancer Research's The Science of Cancer Health Disparities in Racial/Ethnic Minorities and the Medically Underserved Poster Presentation (2019); Abstract Title: "Age-related Differences in Breast Cancer Mortality according to Race/Ethnicity, Insurance, and Socioeconomic Status"; Authors: San Miguel Y, Gomez SL, Murphy J, Schwab RB, McDaniels-Davidson C, Canchola A, Molinolo A, Nodora JN, Martinez ME.

\section{Authors' contributions}

YS assisted in the design of this study, interpretation of the data and was a major contributor in writing and revising this manuscript. SG was a contributor to the conception, design of the study, acquisition and interpretation of the data, and in revising this manuscript. JM contributed to the interpretation of the data and in revising this manuscript. RS contributed to the interpretation of the data and in revising this manuscript. CM contributed to the interpretation of the data and in revising this manuscript. AC contributed to the analysis, interpretation of the data and in writing and revising this manuscript. AM contributed to the interpretation of the data and in revising this manuscript. JN contributed to the interpretation of the data and in revising this manuscript. MM contributed to the conception, design of study, interpretation of the data and in revising this manuscript. All authors read and approved the final manuscript.

\section{Funding}

This work was supported by the Specialized Cancer Center Support Grant to the University of California San Diego Moores Cancer Center (CA023100-29) and by the SDSU/UCSD Comprehensive Cancer Center Partnership (CA132379 and CA132384), which helped fund the analysis, and interpretation of data, and in writing the manuscript. The collection of cancer incidence data used in this study was supported by the California Department of Public Health as part of the statewide cancer reporting program mandated by California Health and Safety Code Section 103885; the National Cancer Institute's Surveillance, Epidemiology and End Results Program under contract HHSN261201000140C awarded to the Cancer Prevention Institute of California, contract HHSN261201000035C awarded to the University of Southern California, and contract HHSN261201000034C awarded to the Public Health Institute; and the Centers for Disease Control and Prevention's National Program of Cancer Registries, under agreement U58DP003862-01 awarded to the California Department of Public Health.

\section{Availability of data and materials}

The datasets generated and/or analyzed during the current study are available upon request to the California Cancer Registry [ccrcal.ca.gov].

\section{Ethics approval and consent to participate}

All procedures performed in this study were approved by the institutional review boards at each institution and in accordance with the ethical standards of the institutions and with the 1964 Helsinki declaration and its later amendments or comparable ethical standards. Consent for this project was waived by the Greater Bay Area Cancer Registry IRB at University of California San Francisco and the IRB at the University of California San Diego. Human subjects' approval was obtained from the UCSF IRB, as a part of the Greater Bay Area Cancer Registry protocol for operating a population-based cancer registry and conducting surveillance and related analyses with the data. Additionally, human subjects' approval was obtained from the UCSD IRB for operating a population-based cancer registry and conducting surveillance and related analyses with the data. The data were anonymized before analysis.

\section{Consent for publication}

Not Applicable.

\section{Competing interests}

Yazmin San Miguel declares that she has no conflict of interest. Author Scarlett Lin Gomez declares that she has no conflict of interest. Author James D. Murphy serves a consultant/advisory role at Boston Consulting Group. Author Richard B. Schwab has stock ownership in Samumed Inc. and serves as an expert witness at Puma. Author Corinne McDaniels-Davidson declares that she has no conflict of interest. Author Alison J. Canchola declares that she has no conflict of interest. Author Alfredo A. Molinolo declares that he has no conflict of interest. Author Jesse N. Nodora declares that he has no conflict of interest. Author Maria Elena Martinez declares that she has no conflict of interest.

\section{Author details}

${ }^{1}$ Moores Cancer Center, University of California San Diego, La Jolla, CA, USA. ${ }^{2}$ Department of Epidemiology and Biostatistics, University of California San Francisco, San Francisco, CA, USA. ${ }^{3}$ Helen Diller Family Comprehensive Cancer Center, University of California San Francisco, San Francisco, CA, USA. ${ }^{4}$ San Diego State University Institute for Public Health, San Diego, CA, USA. ${ }^{5}$ Department of Family Medicine and Public Health, University of California San Diego, La Jolla, CA, USA.

Received: 2 July 2019 Accepted: 28 February 2020

Published online: 17 March 2020

\section{References}

1. American Cancer Society. Breast cancer facts \& figures 2017-2018. Atlanta: American Cancer Society, Inc; 2017

2. Surveillance Research Program, National Cancer Institute SEER*Stat software. 8.3.5 ed. 2018

3. Anders CK, Fan C, Parker JS, Carey LA, Blackwell KL, Klauber-DeMore N, et al, Breast carcinomas arising at a young age: unique biology or a surrogate for aggressive intrinsic subtypes? J Clin Oncol. 2011;29(1):e18-20.

4. Xiong Q, Valero V, Kau V, Kau SW, Taylor S, Smith TL, et al. Female patients with breast carcinoma age 30 years and younger have a poor prognosis: the M.D. Anderson Cancer Center experience. Cancer. 2001;92(10):2523-8.

5. Kothari AS, Beechey-Newman N, D'Arrigo C, Hanby AM, Ryder K, Hamed H, et al. Breast carcinoma in women age 25 years or less. Cancer. 2002;94(3): 606-14.

6. Fredholm H, Eaker S, Frisell J, Holmberg L, Fredriksson I, Lindman H. Breast cancer in young women: poor survival despite intensive treatment. PLoS One. 2009:4(11):e7695.

7. Maggard MA, O'Connell JB, Lane KE, Liu JH, Etzioni DA, Ko CY. Do young breast cancer patients have worse outcomes? J Surg Res. 2003;113(1):109-13.

8. Gnerlich JL, Deshpande AD, Jeffe DB, Sweet A, White N, Margenthaler JA. Elevated breast cancer mortality in women younger than age 40 years compared with older women is attributed to poorer survival in early-stage disease. J Am Coll Surg. 2009;208(3):341-7.

9. Zabicki K, Colbert JA, Dominguez FJ, Gadd MA, Hughes KS, Jones JL, et al. Breast cancer diagnosis in women $<$ or $=40$ versus 50 to 60 years: increasing size and stage disparity compared with older women over time. Ann Surg Oncol. 2006;13(8):1072-7.

10. Reeder-Hayes KE, Wheeler SB, Mayer DK. Health disparities across the breast cancer continuum. Semin Oncol Nurs. 2015;31(2):170-7. 
11. Dunn BK, Agurs-Collins T, Browne D, Lubet R, Johnson KA. Health disparities in breast cancer: biology meets socioeconomic status. Breast Cancer Res Treat. 2010;121(2):281-92.

12. Bernardi D, Errante D, Tirelli U, Salvagno L, Bianco A, Fentiman IS. Insight into the treatment of cancer in older patients: developments in the last decade. Cancer Treat Rev. 2006;32(4):277-88.

13. Bouchardy C, Rapiti E, Fioretta G, Laissue P, Neyroud-Caspar I, Schafer P, et al. Undertreatment strongly decreases prognosis of breast cancer in elderly women. J Clin Oncol. 2003;21(19):3580-7.

14. Deshpande AD, Jeffe DB, Gnerlich J, lqbal AZ, Thummalakunta A, Margenthaler JA. Racial disparities in breast cancer survival: an analysis by age and stage. J Surg Res. 2009;153(1):105-13.

15. Du XL, Fang S, Meyer TE. Impact of treatment and socioeconomic status on racial disparities in survival among older women with breast cancer. Am J Clin Oncol. 2008;31(2):125-32

16. Kim J, Durden E. Socioeconomic status and age trajectories of health. Soc Sci Med. 2007;65(12):2489-502.

17. Osborne C, Ostir GV, Du X, Peek MK, Goodwin JS. The influence of marital status on the stage at diagnosis, treatment, and survival of older women with breast cancer. Breast Cancer Res Treat. 2005;93(1):41-7.

18. Yost K, Perkins C, Cohen R, Morris C, Wright W. Socioeconomic status and breast cancer incidence in California for different race/ethnic groups. Cancer Causes Control. 2001;12(8):703-11.

19. Yang J, Schupp CW, Harrati A, Clarke C, Keegan THM, Gomez SL. Developing an area-based socioeconomic measure from American community survey data. Fremont: Cancer Prevention Institute of California; 2014

20. Ribnikar D, Ribeiro JM, Pinto D, Sousa B, Pinto AC, Gomes E, et al. Breast cancer under age 40: a different approach. Curr Treat Options in Oncol. 2015;16(4):16.

21. DeSantis CE, Ma J, Goding Sauer A, Newman LA, Jemal A. Breast cancer statistics, 2017, racial disparity in mortality by state. CA Cancer J Clin. 2017; 67(6):439-48.

22. Tao L, Gomez SL, Keegan TH, Kurian AW, Clarke CA. Breast cancer mortality in African-American and non-Hispanic White women by molecular subtype and stage at diagnosis: a population-based study. Cancer Epidemiol Biomark Prev. 2015;24(7):1039-45.

23. Ellis L, Canchola AJ, Spiegel D, Ladabaum U, Haile R, Gomez SL. Trends in Cancer survival by health insurance status in California from 1997 to 2014 JAMA Oncol. 2018:4(3):317-23.

24. Hsu CD, Wang X, Habif DV Jr, Ma CX, Johnson KJ. Breast cancer stage variation and survival in association with insurance status and sociodemographic factors in US women 18 to 64 years old. Cancer. 2017; 123(16):3125-31.

25. Azzopardi J, Walsh D, Chong C, Taylor C. Surgical treatment for women with breast cancer in relation to socioeconomic and insurance status. Breast J. 2014;20(1):3-8.

26. Foley KL, Kimmick G, Camacho F, Levine EA, Balkrishnan R, Anderson R. Survival disadvantage among Medicaid-insured breast cancer patients treated with breast conserving surgery without radiation therapy. Breast Cancer Res Treat. 2007:101(2):207-14.

27. Ward E, Halpern M, Schrag N, Cokkinides V, DeSantis C, Bandi P, et al. Association of insurance with cancer care utilization and outcomes. CA Cancer J Clin. 2008;58(1):9-31

28. Giordano SH, Kuo YF, Duan Z, Hortobagyi GN, Freeman J, Goodwin JS. Limits of observational data in determining outcomes from cancer therapy. Cancer. 2008;112(11):2456-66.

\section{Publisher's Note}

Springer Nature remains neutral with regard to jurisdictional claims in published maps and institutional affiliations.

Ready to submit your research? Choose BMC and benefit from:

- fast, convenient online submission

- thorough peer review by experienced researchers in your field

- rapid publication on acceptance

- support for research data, including large and complex data types

- gold Open Access which fosters wider collaboration and increased citations

- maximum visibility for your research: over $100 \mathrm{M}$ website views per year

At $\mathrm{BMC}$, research is always in progress.

Learn more biomedcentral.com/submissions 